\title{
Trials directly comparing alternative spontaneous breathing trial techniques: a systematic review and meta-analysis
}

\author{
Karen E. A. Burns 1,2,3, Ibrahim Soliman 1,2, Neill K. J. Adhikari2, ${ }^{2,4}$, Amer Zwein, ${ }^{1,2}$, Jessica T. Y. Wong ${ }^{5}$, \\ Carolina Gomez-Builes ${ }^{1,2}$, Jose Augusto Pellegrini ${ }^{6,7}$, Lu Chen ${ }^{1,2}$, Nuttapol Rittayamai ${ }^{1,2}$, Michael Sklar ${ }^{1,2}$, \\ Laurent J. Brochard ${ }^{1,2}$ and Jan O. Friedrich ${ }^{1,2^{*}}$
}

\begin{abstract}
Background: The effect of alternative spontaneous breathing trial (SBT) techniques on extubation success and other clinically important outcomes is uncertain.

Methods: We searched MEDLINE, EMBASE, CENTRAL, CINAHL, Evidence-Based Medicine Reviews, Ovid Health Star, proceedings of five conferences (1990-2016), and reference lists for randomized trials comparing SBT techniques in intubated adults or children. Primary outcomes were initial SBT success, extubation success, or reintubation. Two reviewers independently screened citations, assessed trial quality, and abstracted data.

Results: We identified 31 trials ( $n=3541$ patients). Moderate-quality evidence showed that patients undergoing pressure support (PS) compared with T-piece SBTs (nine trials, $n=1901$ ) were as likely to pass an initial SBT (risk ratio (RR) $1.00,95 \%$ confidence interval $(C I) 0.89-1.11 ; l^{2}=77 \%$ ) but more likely to be ultimately extubated successfully (RR 1.06, 95\% Cl 1.02-1.10; 11 trials, $n=1904 ; P^{2}=0 \%$ ). Exclusion of one trial with inconsistent results for SBT and extubation outcomes suggested that PS (vs T-piece) SBTs also improved initial SBT success (RR 1.06, 95\% Cl 1.01-1.12; $P=0 \%$ ). Limited data suggest that automatic tube compensation plus continuous positive airway pressure (CPAP) vs CPAP alone or PS increase SBT but not extubation success.
\end{abstract}

Conclusions: Patients undergoing PS (vs T-piece) SBTs appear to be $6 \%(95 \% \mathrm{Cl} 2-10 \%)$ more likely to be extubated successfully and, if the results of an outlier trial are excluded, $6 \%(95 \% \mathrm{Cl} 1-12 \%)$ more likely to pass an SBT. Future trials should investigate patients for whom SBT and extubation outcomes are uncertain and compare techniques that maximize differences in support.

Keywords: Weaning, Spontaneous breathing trial, Extubation, Systematic review, Meta-analysis, Extubation outcome

\section{Background}

Weaning accounts for approximately $40 \%$ of the time spent on mechanical ventilation $[1,2]$. Compared with nonprotocolized care, randomized controlled trials (RCTs) and a systematic review indicate that weaning protocols reduce the duration of mechanical ventilation, weaning time, and intensive care unit (ICU) length of stay (LOS)

\footnotetext{
* Correspondence: friedrichj@smh.ca

'St Michael's Hospital and the Keenan Research Centre/Li Ka Shing Knowledge Institute, 30 Bond Street, Toronto, ON M5B 1W8, Canada ${ }^{2}$ Interdepartmental Division of Critical Care, University of Toronto, Toronto, ON, Canada

Full list of author information is available at the end of the article
}

$[3,4]$. After identification, patients may undergo a spontaneous breathing trial (SBT) to assess their ability to breathe spontaneously with minimal or no support.

Clinicians conduct SBTs to facilitate decision-making regarding timely extubation and to minimize patients' exposure to invasive ventilation. In making decisions, clinicians 'trade off' the risks associated with delayed extubation and those associated with a premature failed attempt at extubation. Several techniques are commonly used to conduct SBTs, including pressure support (PS) with or without positive end-expiratory pressure (PEEP), continuous positive airway pressure (CPAP), automatic 
tube compensation (ATC), and the T-piece. Whereas some SBT techniques deliver pressure during inspiration to overcome endotracheal tube resistance (e.g., PS, ATC), other techniques aim to improve respiratory mechanics or cardiac function (e.g., CPAP) and may overestimate patients' ability to breathe autonomously after extubation [5]. Conversely, the T-piece provides no support, is perceived to increase work of breathing (WOB), and may underestimate patients' ability to breathe spontaneously after extubation [5]. The most recent American College of Chest Physicians/American Thoracic Society Clinical Practice Guidelines [6] support that an SBT is the major diagnostic test to determine whether patients can be extubated but give only a conditional (weak) recommendation that PS SBTs should be used as the initial SBT, based on limited data (three or four included RCTs).

A Cochrane review of nine trials compared PS and T-piece 'weaning' in adults and found nonsignificant differences between techniques on weaning success, pneumonia, reintubation, ICU mortality, and LOS. In a subgroup analysis (four trials, $n=940$ ) the authors noted that patients were significantly more likely to pass a PS SBT vs a T-piece SBT (risk ratio (RR) 1.09, 95\% confidence interval (CI) 1.02-1.17) [7]. This review did not directly compare alternative SBT techniques and was limited to full publications of adults. At present, no SBT technique has been shown to be superior to another.

We sought to summarize the RCT evidence directly comparing all alternative SBT techniques involving critically ill adults and children on initial SBT success, extubation success, reintubation rate (primary outcomes), and other important outcomes.

\section{Methods}

\section{Data sources}

We searched MEDLINE (1966-February 2017), EMBASE (1980-February 2017), the Cochrane Central Register of Controlled Trials (CENTRAL, February 2017), CINAHL (1982-February 2017), evidence-based medicine reviews, and Ovid Health Star (1999-February 2017) to identify potentially eligible trials using database-specific search strategies without language restrictions. We used the optimally sensitive search strategies of The Cochrane Collaboration for MEDLINE and EMBASE [8-10]. Two authors (KEAB, JOF) independently screened citation titles and abstracts and evaluated full-text versions of potentially relevant trials. Five authors hand-searched conference proceedings of five scientific meetings from 1990-2016: European Society of Intensive Care Medicine, American College of Chest Physicians (except 1999-2002, unavailable), American Thoracic Society, International Symposium of Intensive Care and Emergency Medicine, and Society of Critical Care Medicine (including 2017 for the latter). Ethics approval was not required.

\section{Study selection}

We included randomized or quasi-randomized trials comparing two or more SBT techniques in critically ill adults or children reporting at least one of initial SBT or extubation outcome (success or failure), reintubation, time to extubation or successful extubation, time to first successful SBT, mortality, ventilator-associated pneumonia (VAP), total duration of ventilation, ICU or hospital LOS, postextubation use of noninvasive ventilation (NIV), or adverse events using authors' definitions. We excluded trials that evaluated: neonatal or tracheostomized patients; SBTs as part of a weaning strategy; automated SBTs (e.g., SmartCare ${ }^{\mathrm{Tu}}$, Intellivent $\left.{ }^{\circledR}\right)$; NIV vs continued invasive ventilation; and SBT conduct vs no SBT. Two authors (KEAB, JOF) independently selected trials meeting inclusion criteria, and another author (LJB) adjudicated differences.

\section{Data extraction and quality assessment}

Two unblinded authors (KEAB, JOF) abstracted data regarding the study risk of bias (RoB) (randomization, allocation concealment, blinded outcomes assessment, completeness of follow-up, selective outcomes reporting, stopping early for benefit) and recorded outcomes, using authors' definitions for reported outcomes, on a standardized form [11]. We evaluated RoB (yes, unclear, no) for each domain. Disagreements were resolved by consensus and arbitration with a third author (LJB).

\section{Data synthesis}

We pooled data across studies using random effects models. We derived summary estimates of RR and mean difference (MD) with 95\% CI for binary and continuous outcomes, respectively, using Review Manager 5.3 (Cochrane Collaboration, Oxford, UK) [12]. We pooled 'initial SBT success' in trials that conducted more than one SBT. We evaluated statistical heterogeneity for each outcome using the $I^{2}$ measure with thresholds of $0-40 \%$ (might not be important), 30-60\% (moderate), 50-90\% (substantial), and $\geq 75 \%$ (considerable) $[13,14]$. We summarized trials based on the techniques compared (e.g., T-piece vs other) (Additional file 1).

We planned subgroup analyses to compare the effects of different techniques on primary outcomes in perioperative vs nonperioperative trials and based on: duration of ventilation at randomization (nonperioperative trials); the support provided during SBTs; the type of lung disease; and methodologic quality (low/moderate vs high RoB). We assessed for subgroup differences using the chi-square test [15].

We used the Grading of Recommendations Assessment, Development and Evaluation (GRADE) system to assess the quality of the body of evidence associated with the primary outcomes and significant secondary outcomes [16]. 
We assessed for publication bias when at least 10 trials were identified [17]. A written protocol was used to guide the review process.

\section{Results}

\section{Trial identification}

We identified 4218 unique citations. We assessed 187 articles for eligibility and excluded 156 studies (Fig. 1). Thirty-one trials [18-48] reporting on 3541 patients met our inclusion criteria including five trials comparing three SBT techniques [23, 24, 29, 30, 48]. Two trials $[38,46]$ appeared to be published, at least in part, in duplicate $[49,50]$. The most common comparisons were T-piece vs PS (13 trials), T-piece vs CPAP (nine trials), PS vs ATC (three trials), and CPAP vs ATC (three trials). Four trials $[21,34,38,40]$ were not published in English. Six trials $[25,27,33,36,45,47]$ were published as abstracts, of which two [36, 47] provided partial or full-text manuscripts. Nine trials [19-21, 23-25, 44, 45, 47] evaluated perioperative populations (six cardiac surgical [19-21, 24, $25,44]$ and three other surgical $[23,45,47])$. Three trials $[28,43,46]$ evaluated pediatric patients.

\section{Quality assessment}

We judged randomization and allocation concealment to be at low RoB in 16 (52\%) trials and 17 (55\%) trials, respectively. One quasi-randomized trial allocated patients based on even or odd days [34]. No trial evaluated outcomes in a blinded manner. We judged 15 (48\%) trials to have complete outcomes reporting. Eighteen (58\%) trials conducted an intention-to-treat analysis and 26 (84\%) trials did not stop early for benefit. Overall trial quality was moderate (Additional file 2: Figure S1).

\section{Primary outcomes \\ Initial SBT success}

Seventeen T-piece, 12 CPAP, eight ATC, and 13 PS trials directly compared one SBT technique with another and reported initial SBT success (Table 1). Compared with T-piece SBTs, moderate-quality evidence supports that patients undergoing PS SBTs were not more likely to pass an SBT (RR 1.00, 95\% CI 0.89-1.11; $p=1.0$; nine trials, $n=1901)$ with considerable heterogeneity $\left(I^{2}=77 \%\right)$ (Table 2, Fig. 2, Additional file 3: Figure S2).

Low-quality evidence from three trials $(n=247)$ suggests that patients were significantly more likely to pass an SBT with ATC + CPAP compared with CPAP alone (RR 1.12, 95\% CI 1.04-1.22; $p=0.005, I^{2}=0 \%$ ). Similarly, low-quality evidence from three trials $(n=276)$ showed that patients were significantly more likely to pass an SBT with ATC + CPAP compared with PS (RR 1.10, 95\% CI 1.01-1.20; $p=0.02, I^{2}=0 \%$ ) (Table 3).

\section{Extubation success}

Seventeen T-piece, eight CPAP, eight ATC, and 14 PS trials compared one SBT technique with another and reported extubation success (Table 1). Moderate-quality evidence supports that patients undergoing PS compared

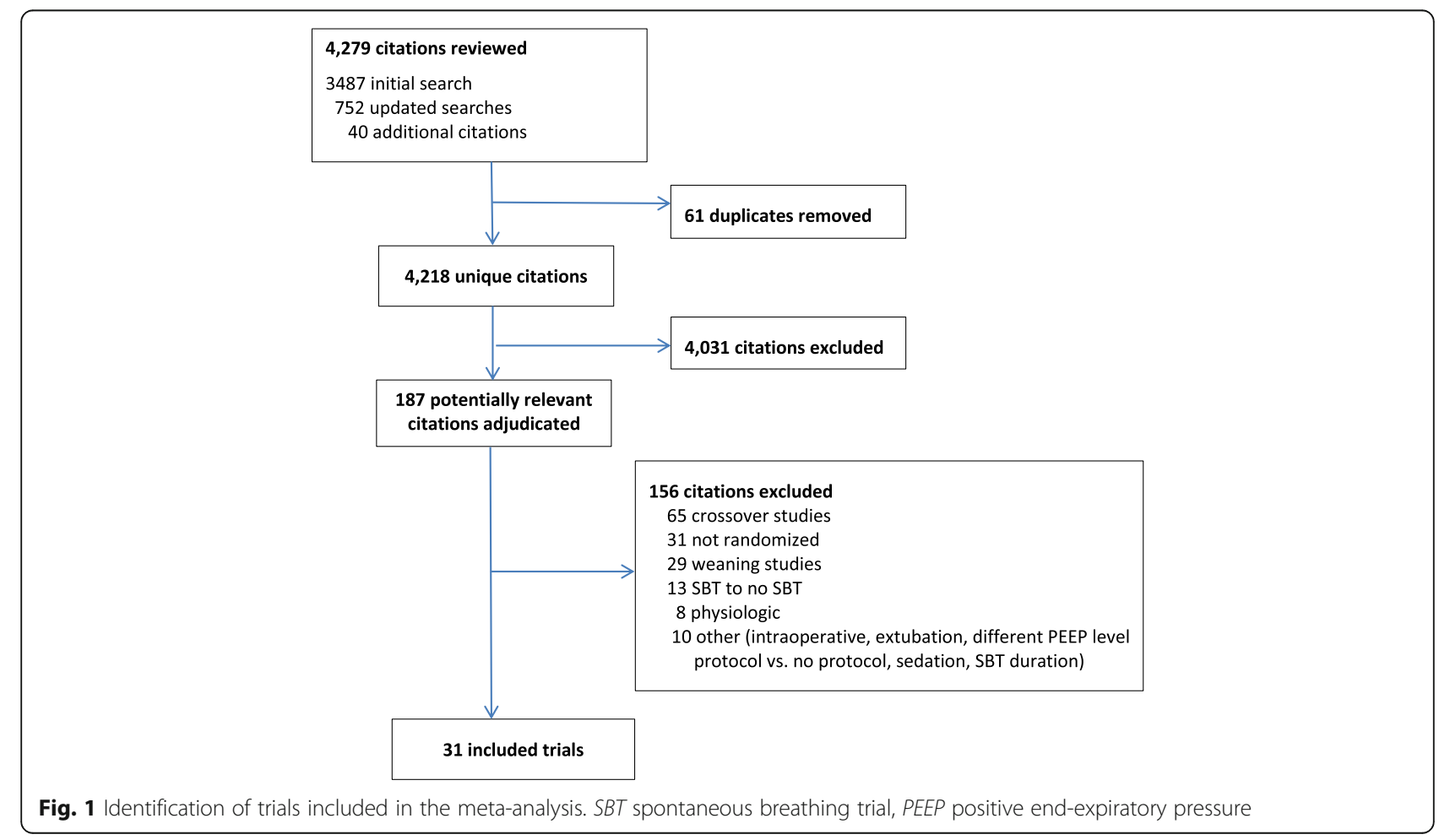


Table 1 Characteristics of included trials

\begin{tabular}{|c|c|c|c|c|c|}
\hline Author and year & Interventions & Country & $\begin{array}{l}\text { Publication } \\
\text { Type }\end{array}$ & Population & $\begin{array}{l}\text { Duration of ventilation } \\
\text { at inclusion }\end{array}$ \\
\hline $\begin{array}{l}\text { Feeley } 1975[18] \\
(n=25)\end{array}$ & T-piece/PEEP $5 \mathrm{cmH}_{2} \mathrm{O}$ vs T-piece & USA & Full & Adult & Not reported \\
\hline $\begin{array}{l}\text { Hastings } 1980[19] \\
(n=18)\end{array}$ & IMV/CPAP $5 \mathrm{cmH}_{2} \mathrm{O}$ vs T-piece/CPAP $5 \mathrm{cmH}_{2} \mathrm{O}$ & USA & Full & Adult & Perioperative \\
\hline $\begin{array}{l}\text { Prakash } 1982[20] \\
(n=28)\end{array}$ & IMV vs SVT (on ventilator) & The Netherlands & Full & Adult & Perioperative \\
\hline $\begin{array}{l}\text { Koller } 1983[21] \\
(n=45)\end{array}$ & CPAP $10 \mathrm{cmH}_{2} \mathrm{O}$ vs T-piece/ZEEP & Austria & Full & Adult & Perioperative \\
\hline $\begin{array}{l}\text { Jones } 1991[22] \\
(n=106)\end{array}$ & CPAP $5 \mathrm{cmH}_{2} \mathrm{O}$ vs T-piece/ZEEP & USA & Full & Adult & Not reported \\
\hline $\begin{array}{l}\text { Abalos } 1992[23] \\
(n=62)\end{array}$ & SIMV vs CPAP $4 \mathrm{cmH}_{2} \mathrm{O}$ vs T-piece & USA & Full & Adult & Perioperative \\
\hline $\begin{array}{l}\text { Bailey } 1995[24] \\
(n=82)\end{array}$ & T-piece ${ }^{\mathrm{a}}$ vs CPAP $5 \mathrm{cmH}_{2} \mathrm{O}$ vs CPAP $10 \mathrm{cmH}_{2} \mathrm{O}$ & England & Full & Adult & Perioperative \\
\hline $\begin{array}{l}\text { Schinco } 1995[25] \\
(n=30)\end{array}$ & PS $5 \mathrm{cmH}_{2} \mathrm{O} / \mathrm{CPAP} 5 \mathrm{cmH}_{2} \mathrm{O}$ vs CPAP $5 \mathrm{cmH}_{2} \mathrm{O}$ & USA & Abstract & Adult & Perioperative \\
\hline $\begin{array}{l}\text { Esteban } 1997[26] \\
(n=484)\end{array}$ & T-piece vs PS $7 \mathrm{cmH}_{2} \mathrm{O}$ & $\begin{array}{l}\text { Spain and South } \\
\text { America }\end{array}$ & Full & Adult & $>48$ hours \\
\hline $\begin{array}{l}\text { Holanda } 2000[27] \\
(n=35)\end{array}$ & T-piece vs PS & Brazil & Abstract & Adult & $>48$ hours \\
\hline $\begin{array}{l}\text { Farias } 2001[28] \\
(n=257)\end{array}$ & T-piece vs PS $10 \mathrm{cmH}_{2} \mathrm{O} \pm$ PEEP $5 \mathrm{cmH}_{2} \mathrm{O}$ & Argentina & Full & Pediatric & $>48$ hours \\
\hline $\begin{array}{l}\text { Haberthur } 2002[29] \\
(n=90)\end{array}$ & $\begin{array}{l}\text { PS } 5 \mathrm{cmH}_{2} \mathrm{O} / \text { PEEP } 5 \mathrm{cmH}_{2} \mathrm{O} \text { vs ATC/PEEP } 5 \\
\mathrm{cmH}_{2} \mathrm{O} \text { vs T-piece }\end{array}$ & Switzerland & Full & Adult & $>24$ hours \\
\hline $\begin{array}{l}\text { Koksal } 2004[30] \\
(n=60)\end{array}$ & $\begin{array}{l}\mathrm{PS} \leq 10 \mathrm{cmH}_{2} \mathrm{O} / \mathrm{PEEP} \leq 5 \mathrm{cmH}_{2} \mathrm{O} \text { vs } \mathrm{CPAP} \leq 5 \\
\mathrm{cmH}_{2} \mathrm{O} \text { vs T-piece }\end{array}$ & Turkey & Full & Adult & $>48$ hours \\
\hline $\begin{array}{l}\text { Matic } 2004[31] \\
(n=260)\end{array}$ & T-piece vs PS $8 \mathrm{cmH}_{2} \mathrm{O}$ & Croatia & Full & Adult & $>48$ hours \\
\hline $\begin{array}{l}\text { Cohen } 2006[32] \\
(n=99)\end{array}$ & $\mathrm{ATC}^{\mathrm{b}} / \mathrm{CPAP} 5 \mathrm{cmH}_{2} \mathrm{O}$ vs CPAP $5 \mathrm{cmH}_{2} \mathrm{O}$ & Israel & Full & Adult & $>24$ hours \\
\hline $\begin{array}{l}\text { Liang } 2006[33] \\
(n=97)\end{array}$ & ATC vs T-piece & Taiwan & Abstract & Adult & $>4$ days \\
\hline $\begin{array}{l}\text { Colombo } 2007[34] \\
(n=120)\end{array}$ & T-piece vs PS $7 \mathrm{cmH}_{2} \mathrm{O} / \mathrm{PEEP} 5 \mathrm{cmH}_{2} \mathrm{O}$ & Brazil & Full & Adult & $>48$ hours \\
\hline $\begin{array}{l}\text { Matic } 2007[35] \\
(n=136)\end{array}$ & T-piece vs PS (not specified) & Croatia & Full & Adult & $>24$ hours \\
\hline $\begin{array}{l}\text { Fayed } 2008[36] \\
(n=30)\end{array}$ & ATC $/$ CPAP $5 \mathrm{cmH}_{2} \mathrm{O}$ vs CPAP $5 \mathrm{cmH}_{2} \mathrm{O}$ & Egypt & Abstract & Adult & $>24$ hours \\
\hline $\begin{array}{l}\text { Cohen } 2009[37] \\
(n=180)\end{array}$ & $\begin{array}{l}\text { ATC } / \text { /CPAP } 5 \mathrm{cmH}_{2} \mathrm{O} \text { vs PS } 7 \mathrm{cmH}_{2} \mathrm{O} / \mathrm{CPAP} \\
5 \mathrm{cmH}_{2} \mathrm{O}\end{array}$ & Israel & Full & Adult & $>24$ hours \\
\hline $\begin{array}{l}\text { Zhang } 2009[38] \\
(n=208)\end{array}$ & T-piece vs PS $5 \mathrm{cmH}_{2} \mathrm{O} / \mathrm{PEEP} 5 \mathrm{cmH}_{2} \mathrm{O}$ & China & Full & Adult & Not reported \\
\hline $\begin{array}{l}\text { Figueroa-Casas } 2010[39] \\
(n=122)\end{array}$ & ATC $^{\mathrm{b}} / \mathrm{PEEP} 5 \mathrm{cmH}_{2} \mathrm{O}$ vs CPAP $5 \mathrm{cmH}_{2} \mathrm{O}$ & USA & Full & Adult & $>24$ hours \\
\hline $\begin{array}{l}\text { Molina-Saldarriaga } 2010 \text { [40] } \\
(n=50)\end{array}$ & CPAPC vs T-piece & Colombia & Full & Adult & $>48$ hours \\
\hline $\begin{array}{l}\text { Cekman } 2011[41] \\
(n=40)\end{array}$ & $\mathrm{CPAP} \leq 5 \mathrm{cmH}_{2} \mathrm{O}$ vs T-piece & Turkey & Full & Adult & $>48$ hours \\
\hline $\begin{array}{l}\text { Vats 2012 [42] } \\
(n=40)\end{array}$ & T-piece vs PS $7 \mathrm{cmH}_{2} \mathrm{O}$ & India & Full & Adult & Not reported \\
\hline $\begin{array}{l}\text { El-beleidy } 2013[43] \\
(n=36)\end{array}$ & $\begin{array}{l}\text { ATC } / \text { CPAP } 5 \mathrm{cmH}_{2} \mathrm{O} \text { vs PS } 6-10 \mathrm{cmH}_{2} \mathrm{O} / \mathrm{CPAP} \\
5 \mathrm{cmH}_{2} \mathrm{O}\end{array}$ & Egypt & Full & Pediatric & $>24$ hours \\
\hline
\end{tabular}


Table 1 Characteristics of included trials (Continued)

\begin{tabular}{|c|c|c|c|c|c|}
\hline $\begin{array}{l}\text { Lourenco } 2013[44] \\
(n=30)\end{array}$ & T-piece vs PS (not specified) & Brazil & Full & Adult & Perioperative \\
\hline $\begin{array}{l}\text { Sherif } 2013[45] \\
(n=100)\end{array}$ & PS (not specified) vs PS/ATC & Egypt & Abstract & Adult & Not reported \\
\hline $\begin{array}{l}\text { Bilan } 2015[46] \\
(n=51)\end{array}$ & CPAP vs T-piece & Iran & Full & Pediatric & Not reported \\
\hline $\begin{array}{l}\text { Chittawatanarat } 2015[47] \\
(n=520)\end{array}$ & T-piece vs PS $7 \mathrm{cmH}_{2} \mathrm{O} / \mathrm{PEEP} \leq 5 \mathrm{cmH}_{2} \mathrm{O}$ & Thailand & Abstract & Adult & $>12$ hours \\
\hline $\begin{array}{l}\text { Teixeira } 2015[48] \\
(n=160)\end{array}$ & $\begin{array}{l}\text { PS } 7 \mathrm{cmH}_{2} \mathrm{O} / \mathrm{PEEP} 5-8 \mathrm{cmH}_{2} \mathrm{O} \text { vs PAV+/PEEP } \\
5-8 \mathrm{cmH}_{2} \mathrm{O} \text { vs T-piece }\end{array}$ & Brazil & Full & Adult & $>24$ hours \\
\hline
\end{tabular}

PEEP positive end-expiratory pressure, IMV intermittent mandatory ventilation, CPAP continuous positive airway pressure, SVT spontaneous ventilation trial, ZEEP zero end-expiratory pressure, SIMV synchronized intermittent mandatory ventilation, $P S$ pressure support, $A T C$ automatic tube compensation; $P A V+$ proportional assist ventilation with load adjustable gain factors

${ }^{\mathrm{a}} \mathrm{T}$-piece with CPAP $\mathrm{O} \mathrm{cmH}_{2} \mathrm{O}$

${ }^{\mathrm{b}}$ ATC with $100 \%$ compensation

${ }^{c}$ CPAP set to $85 \%$ of intrinsic PEEP

with T-piece SBTs were significantly more likely to be extubated successfully (RR 1.06, 95\% CI 1.02-1.10; $p=0.007 ; 11$ trials, $n=1904 ; I^{2}=0 \%$ ) (Table 2, Fig. 3, Additional file 4: Figure S3).

\section{Reintubation rate}

Fourteen T-piece, nine CPAP, seven ATC, and 13 PS trials comparing one SBT technique with another reported the reintubation rate and found no statistically significant differences between techniques (Table 1, Additional file 5 : Figure S4).

\section{Secondary outcomes}

There was no effect of one SBT technique vs another on ICU mortality (seven T-piece, three CPAP, and five PS trials), hospital mortality (four T-piece and four PS trials), or the most protracted mortality measure (10 T-piece, four CPAP, and seven PS trials).

No trial reported time to extubation or time to successful extubation. Meta-analysis of three trials comparing ATC + CPAP with CPAP alone found no difference in NIV use after extubation (RR 0.53, 95\% CI 0.27-1.06; $p=0.07, I^{2}=0 \%$ ).

\section{Sensitivity, subgroup, and post-hoc analyses}

Exclusion of a single quasi-randomized trial comparing PS vs T-piece SBTs [34] did not change the significant increase in extubation success favoring PS SBTs (RR 1.05, 95\% CI 1.01-1.10; $p=0.02, I^{2}=0 \%$ ).

Meta-analyses of PS vs T-piece SBTs showed benefit in seven nonperioperative trials $(n=1273$; RR $1.07,95 \% \mathrm{CI}$ $\left.1.01-1.13 ; \quad p=0.02, \quad I^{2}=94 \%\right)$ (high-quality evidence) compared with two perioperative trials $(n=548$; RR 0.86 , 95\% CI $0.61-1.22 ; p=0.41, I^{2}=0 \%$ ) (low-quality evidence); however, an interaction test showed no difference between these summary estimates $(p=0.23)$ (Additional file 6: Table S1, Additional file 7: Figure S5). Subgroup analyses based on duration of ventilation among nonperioperative trials was not feasible given similar reported durations of ventilation. Subgroup analyses comparing more vs less inspiratory support and type of lung disease were not significant for commonly reported comparisons of alternative techniques. A sensitivity analysis was not possible due to the absence of blinded outcomes assessment across trials. Inspection of a funnel plot for 11 trials comparing PS with T-piece SBTs on extubation success did not suggest publication bias.

Table 2 Summary of findings: PS vs T-piece SBTs on SBT and extubation success

\begin{tabular}{|c|c|c|c|c|c|}
\hline \multirow[t]{2}{*}{ Outcome } & \multicolumn{2}{|c|}{ Illustrative comparative risks ${ }^{a}(95 \% \mathrm{Cl})$} & \multirow{2}{*}{$\begin{array}{l}\text { Risk ratio } \\
(95 \% \mathrm{Cl})\end{array}$} & \multirow{2}{*}{$\begin{array}{l}\text { Number of } \\
\text { participants } \\
\text { (trials) }\end{array}$} & \multirow{2}{*}{$\begin{array}{l}\text { Quality of evidence } \\
\text { (GRADE) }\end{array}$} \\
\hline & Assumed risk, T-piece & Corresponding risk, pressure support & & & \\
\hline \multirow{2}{*}{$\begin{array}{l}\text { PS vs T-piece SBTs } \\
\text { on SBT success }\end{array}$} & Study population & & $1.00(0.89-1.11)$ & 1901 (9 trials) & $\oplus \oplus \oplus \ominus$ moderate $^{b}$ \\
\hline & 766 per 1000 & 766 per $1000(681-850)$ & & & \\
\hline \multirow{2}{*}{$\begin{array}{l}\text { PS vs T-piece SBTs on } \\
\text { extubation success }\end{array}$} & Study population & & $1.06(1.02-1.1)$ & 1904 (11 trials) & $\oplus \oplus \oplus \ominus$ moderate ${ }^{c}$ \\
\hline & 749 per 1000 & $794^{d}$ per $1000(764-824)$ & & & \\
\hline
\end{tabular}

PS pressure support, $C l$ confidence interval, SBT spontaneous breathing trial, GRADE Grading of Recommendations Assessment, Development and Evaluation, NNT number needed to treat

${ }^{a}$ The assumed risk is based on the median control group risk across studies. The corresponding risk (and its $95 \% \mathrm{Cl}$ ) is based on the assumed risk in the comparison group and the relative effect of the intervention (and its $95 \% \mathrm{Cl}$ ). NNT is $1000 /(794-749)=22(95 \% \mathrm{Cl} 13-67)$

${ }^{\mathrm{b}}$ The Chittawatanarat [47] trial skews data, increases heterogeneity, and changes summary estimate of effect. It also changes our interpretation of the findings

${ }^{\mathrm{c}}$ Methodologic concerns with the Colombo trial (quasi-randomized) [34]; this trial carries 10\% weight in the pooled extubation outcome meta-analysis

${ }^{\mathrm{d} C}$ Corresponds to NNT of $794-749=45$ or $1000 / 45=22$ (95\% Cl 13-67) 


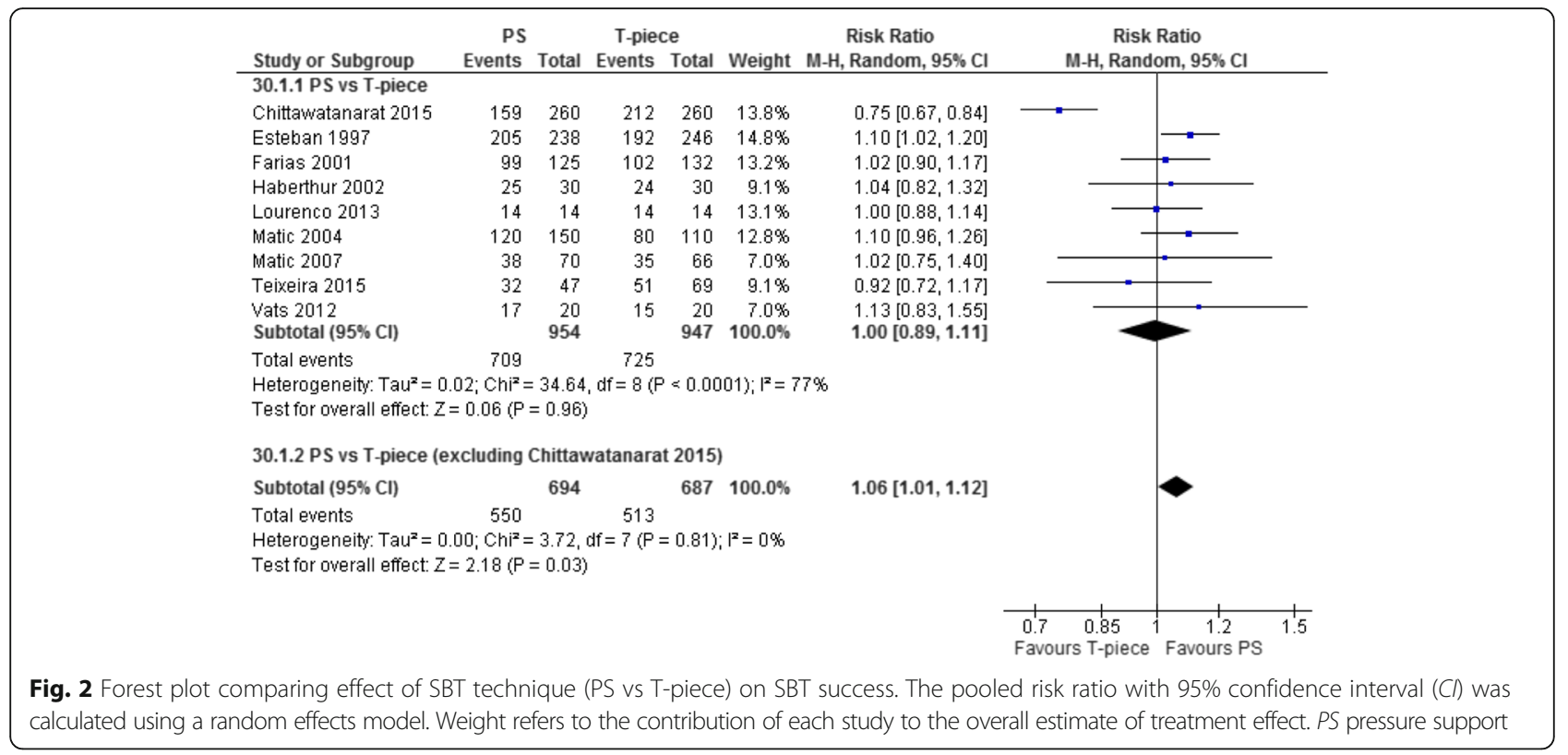

We conducted a post-hoc analysis that excluded a single, surgical trial [47] which enrolled surgical patients, was published in abstract form only, and had internally inconsistent results (i.e., lower initial SBT success rate but higher extubation success rate for PS vs T-piece SBTs). When this trial was excluded, meta-analyses showed that more patients passed an initial PS (vs Tpiece) SBT (RR 1.06, 95\% CI 1.01-1.12; $p=0.03$ ) without heterogeneity $\left(I^{2}=0 \%\right)$ and were similarly extubated successfully (RR 1.06, 95\% CI 1.01-1.12; $p=0.03, I^{2}=0 \%$ ). Excluding each of the other RCTs in a full leave-onestudy-out analysis did not result in significant changes to the pooled effect estimate for SBT success (pooled RR range, $0.98-1.01)$ or heterogeneity ( $I^{2}$ range, $\left.77-80 \%\right)$ suggesting that only a single trial [47] was an outlier for this outcome. In a similar analysis for extubation success, the pooled RR ranged from 1.05 to 1.07 and remained statistically significant with no heterogeneity $\left(I^{2}=0 \%\right)$ regardless of which RCT was removed, suggesting that no trial was an outlier or disproportionately influenced the extubation success summary estimate.

Table 3 Summary estimates of effect for comparisons of ATC vs other techniques on SBT success

\begin{tabular}{lllll}
\hline Comparison & Trials & Risk ratio $(95 \%$ Cl $)$ & $p$ value & $P^{2}(\%)$ \\
\hline ATC/CPAP vs CPAP & $3(n=247)$ & $1.12(1.04-1.22)$ & 0.005 & 0 \\
ATC/CPAP vs PS & $3(n=276)$ & $1.10(1.01-1.20)$ & 0.02 & 0 \\
ATC vs T-piece & $2(n=157)$ & $1.03(0.76-1.42)$ & 0.83 & 81 \\
ATC/PS vs PS & $1(n=100)$ & $1.04(0.94-1.15)$ & 0.40 & NA \\
\hline
\end{tabular}

ATC automatic tube compensation, CPAP continuous positive airway pressure, $P S$ pressure support, $\mathrm{Cl}$ confidence interval, $l^{2}$ measure of heterogeneity, SBT spontaneous breathing trial

\section{Discussion}

We identified 31 trials of overall moderate quality reporting on 3541 patients. Moderate-quality evidence supported that SBT success rates were similar with PS and T-piece, with substantial heterogeneity. However, post-hoc exclusion of an unpublished trial [47] with inconsistent results eliminated the heterogeneity and showed that SBT success was 6\% more likely with PS SBTs. Meta-analysis also showed a $6 \%$ higher probability of successful extubation following PS (vs T-piece) SBTs, with no heterogeneity, irrespective of this trial's inclusion [47]. Although a $6 \%$ relative improvement in probability of successful extubation may appear small, it corresponds to a clinically important number needed to treat of 22 (95\% CI 13-67) when the baseline risk of extubation success is $75 \%$ (Table 2). Low-quality evidence from three trials indicated that patients were $12 \%$ more likely to pass an SBT with ATC + CPAP/PEEP compared with CPAP and were $10 \%$ more likely to pass an SBT with ATC + CPAP/PEEP compared with PS, although extubation success rates were similar. We found no differences between alternative SBT techniques on reintubation rate or mortality, although CIs were wide. Subgroup analysis suggested beneficial effects of PS vs T-piece SBTs on SBT success in seven nonperioperative trials (high-quality evidence) compared with two perioperative trials (low-quality evidence), but the RRs were not statistically dissimilar.

Most trials directly compared T-piece with PS SBTs (13 trials) and T-piece with CPAP SBTs (nine trials). Very few trials assessed alternative SBT techniques in children. Most trials $(n=22)$ were conducted in patients who were nonperioperative and for whom extubation 


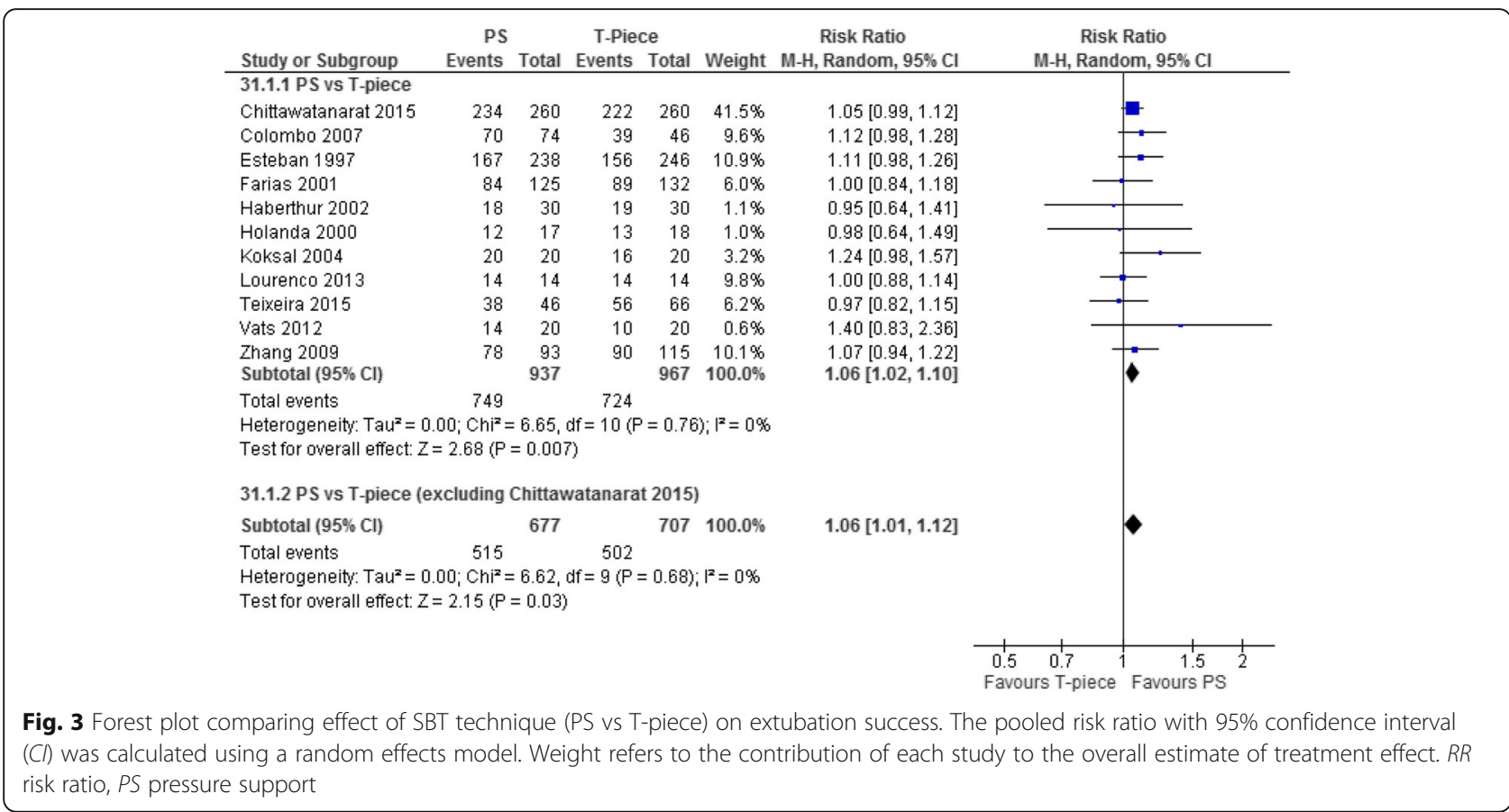

decisions are considered more challenging. In pooling outcomes, we noted that five of six cardiac surgery trials reported a $100 \%$ SBT success rate in both arms and three surgical trials reported a $100 \%$ extubation success rate in both arms. These findings suggest that the most important question in postoperative patients with a high pretest probability of SBT and extubation success may be whether an SBT is necessary and that questions regarding the best SBT technique to use may be most relevant to patients at indeterminate or low pretest probability of success.

Our systematic review differs from two previous reviews by directly comparing SBT techniques and excluding trials evaluating SBT techniques as one component of a weaning strategy $[7,51]$. Moreover, we hand-searched conference proceedings spanning 26 years, where feasible, and included pediatric trials. Compared with the Cochrane review of nine trials [7], we included nine additional trials (one pediatric trial [28], four adult trials [34, 38, 42, 44], two abstracts [27, 47], and two three-arm trials [30,48]) comparing T-piece and PS SBTs and excluded four weaning trials [52-55]. Contrary to their findings, we found that patients were only more likely to pass a PS (vs Tpiece) SBT after exclusion of a single outlier trial [47] but were significantly more likely to be extubated successfully. This finding remained significant after exclusion of a single pediatric trial [28]. Compared with a recent meta-analysis of 12 trials [51], we included five additional trials (one pediatric trial [28], three adult trials [34, 42, 48] including a three-arm trial [48], and an abstract [27]) and excluded four trials involving weaning or tracheostomized patients [52, 53, 55, 56]. Similar to their review, we found that the SBT technique did not influence rates of weaning success, mortality, or reintubation.

Considerable debate exists regarding the SBT technique that best simulates patient's WOB after extubation. An SBT approximates the patient's ability to breathe spontaneously, but it is an imperfect test because it cannot take into consideration factors (e.g., upper airway resistance, respiratory muscle fatigue, cardiac decompensation, accumulation of secretions) that may occur after extubation. There are several physiological reasons for the clinical observation that PS SBTs may lead to more successful initial SBTs and extubations. By overcoming a portion of the pressure gradient across the endotracheal tube, low levels of PS or CPAP provide minimal but potentially important support during an SBT. A systematic review of the effect of different SBTs on physiological outcomes found that metrics of patient effort (WOB $(\mathrm{n}=142)$ and pressure-time product (PTP) $(n=129))$ were significantly higher during T-piece vs PS SBTs, with significant heterogeneity $\left(I^{2} \geq 75 \%\right)$. These metrics during T-piece SBTs were also more comparable with the postextubation period compared with PS SBTs, although sample sizes were small $(n=77$ for WOB; $n=52$ for PTP) and heterogeneity was moderate $\left(I^{2}=67 \%\right.$ and $62 \%$, respectively) [57]. Most patients, especially those with high pretest probability of success who represent the majority of patients submitted to SBTs $[6,58]$, can be extubated easily after an initial SBT [58]. However, T-piece SBTs may be appropriate in selected patients (e.g. severe left ventricular dysfunction, neuromuscular weakness, 
difficult airway) when clinicians are uncertain regarding their ability to breathe on their own and when they prioritize a low false positive rate for passing an SBT and being extubated successfully due to the risks associated with extubation failure [59]. Conversely, when T-piece (vs PS) SBTs are used in patients with a high likelihood of extubation success, they may induce a high false negative rate. Compared with T-piece SBTs, our review may suggest that PS SBTs facilitate extubation decision-making. Even if PS SBTs underestimate postextubation WOB, their successful completion may offset clinician reluctance to extubate, resulting in more timely and successful extubation without increased reintubation $[60,61]$. This may be the primary reason why PS SBTs result in both higher SBT and extubation success rates; otherwise one might expect a test that underestimates postextubation WOB to yield a higher SBT success rate followed by a higher reintubation rate. However, reintubation may be related not only to SBT technique and outcome but also to extubation readiness and new and concomitant illnesses. Furthermore, compared with T-piece SBTs, PS SBTs do not require that clinicians assemble a $\mathrm{T}$-piece circuit or disconnect the patient from the ventilator or its alarms, and permit application of PEEP that reduces the potential for loss of lung aeration immediately prior to extubation. Although passing an initial SBT is an important outcome, patients may undergo serial SBTs before extubation, and stakeholders prioritize being extubated successfully [62].

Several additional findings warrant commentary. First, few trials reported use of daily screening or the criteria used to identify SBT candidates and assess extubation readiness. Second, we noted wide variation in initial SBT success rates across trials comparing PS (range, 54.3100.0\%) with T-piece (range, 53.0-100.0\%) SBTs and comparing ATC + CPAP (range, 64.7-96.7\%) with PS (range, 52.6-86.0\%) SBTs, and, similarly, broad variation in extubation success rates comparing PS (range, 60.0100.0\%) with T-piece (range, 50.0-100.0\%) SBTs. Conversely, we noted higher SBT success rates in three trials comparing ATC and CPAP (range, 93.3-96.6\%) with CPAP alone (range, 80.0-86.7\%). While SBT and extubation summary estimates differed quantitatively across comparisons, qualitatively the direction of effect favored SBTs conducted with inspiratory support. Third, only eight trials [25, 28-30, 34, 37, 38, 43] specified addition of CPAP (or PEEP) to PS during SBTs. Fourth, trials were predominantly of moderate quality. Finally, although we included pediatric trials, we only identified three such trials. Because each pediatric trial compared different techniques (T-piece vs PS + PEEP [28], ATC/ CPAP vs PS/CPAP [43], and CPAP vs T-piece [46]) our ability to compare outcomes in children vs adults was limited. Results for the pediatric trials were not different from the pooled results of the adult trials for any of the outcomes (interaction $p$ values all nonsignificant; results not shown).

Our review is the first to directly compare alternative SBT techniques and was strengthened by an extensive search, duplicate citation screening and data abstraction, use of random effects models to pool data, and conduct of prespecified subgroup analyses. Our review also has limitations. Included trials were predominantly of moderate quality with no trial conducting blinded outcomes assessment and summary estimates were limited by variable outcomes reporting and unclear prospective followup. Statistical noise could be minimized if SBT techniques were applied serially until extubation and if extubation was restricted to patients who passed an SBT. Only five trials, all comparing PS with T-piece SBTs, reported conducting SBTs daily [31, 35], daily up to 3 days [29, 48], or for an undisclosed time [38]. Despite subgroup analyses, we cannot fully elucidate the impact of pretest probability of SBT or extubation success on the effect of SBT as patients at intermediate or high likelihood of SBT and extubation success dominated the analysis. The implications of our findings for patients with low pretest probability remain uncertain. Finally, our ability to assess the impact of other factors (e.g., pre-randomization duration of ventilation, type of ICU, presence of dedicated respiratory care personnel) in subgroup analyses was constrained by small numbers of trials and variable and limited reporting. Furthermore, SBT durations were variable. Two RCTs randomizing patients to $30 \mathrm{vs}$ 120 minutes duration of T-piece [63] or PS [64] SBTs found nonsignificant differences in SBT and extubation success rates. In addition, a very recent multicenter RCT published only in abstract form [65] suggests that reventilating patients for 1 hour after a successful SBT may increase successful extubation rates.

\section{Conclusion}

Patients undergoing PS vs T-piece SBTs appear to be $6 \%$ (95\% CI 2-10\%,) more likely to be extubated successfully, and, if the results of an outlier trial are excluded, are $6 \%$ (95\% CI 1-12\%) more likely to pass an SBT. PS SBTs were not associated with an increased risk of reintubation or mortality, but CIs were wide.

\section{Additional files}

\section{Additional file 1: Additional methods, results, and discussion.} (DOCX $15 \mathrm{~kb}$ )

Additional file 2: Figure S1. Risk of bias for the included trials. (DOCX $30 \mathrm{~kb}$ )

Additional file 3: Figure S2. Forest plot comparing PS vs other techniques on SBT success. (DOCX $41 \mathrm{~kb}$ ) 
Additional file 4: Figure S3. Forest plot comparing PS vs other techniques on extubation success. (DOCX 42 kb)

Additional file 5: Figure S4. Forest plot comparing SBT technique (PS vs other technique) on reintubation. The pooled risk ratio with $95 \%$ $\mathrm{Cl}$ was calculated using a random effects model. Weight refers to the contribution of each study to the overall estimate of treatment effect. (DOCX $40 \mathrm{~kb}$ )

Additional file 6: Table S1. Summary of findings for PS vs T-piece SBTs on SBT success based on pretest probability. (DOCX $16 \mathrm{~kb}$ )

Additional file 7: Figure S5. Subgroup analysis: forest plot comparing PS vs T-piece on SBT success based on pretest probability. (DOCX $30 \mathrm{~kb}$ )

\section{Abbreviations}

ATC: Automatic tube compensation; Cl: Confidence interval; CPAP: Continuous positive airway pressure; GRADE: Grading of Recommendations Assessment, Development and Evaluation; ICU: Intensive care unit; LOS: Length of stay; MD: Mean difference; NIV: Noninvasive ventilation; PEEP: Positive end-expiratory pressure; PS: Pressure support; RCT: Randomized controlled trial; RoB: Risk of bias; RR: Risk ratio; SBT: Spontaneous breathing trial; VAP: Ventilator-associated pneumonia; WOB: Work of breathing

\section{Acknowledgements}

This manuscript was presented in abstract form at the annual assembly of the American Thoracic Society (2016). The authors wish to thank Mr David Lightfoot (librarian, St. Michael's Hospital) for his assistance in conducting the original and updated literature searches.

\section{Funding}

No specific funding was received for this study. At the time of conducting this systematic review, KEAB and JOF held Clinician Scientist-Phase 2 Awards from the Canadian Institutes of Health Research, and KEAB also held an Ontario Ministry of Research and Innovation Early Researcher Award. LJB holds the Keenan Chair in Critical Care and Acute Respiratory Failure (St. Michael's Hospital, Toronto, Canada).

\section{Availability of data and material}

KEAB and JOF had full access to all of the data in the study, take responsibility for the integrity of the data and the accuracy of the data analysis, and will make available the RevMan files used to conduct the analyses.

\section{Authors' contributions}

KEAB and JOF conducted the literature searches, selected studies meeting inclusion criteria, extracted data and assessed study quality, and conducted risk of bias assessments. KEAB, JOF, IS, JAP, LC, NR, and MS screened abstracts. JAP reviewed selected studies published in foreign languages, extracted data and assessed study quality, and assisted with adjudicating whether studies met inclusion criteria. JOF also reviewed selected foreign language publications. IS, AZ, NKJA, JTYW, and CG-B searched conference proceedings. IS and KEAB adjudicated abstracts for inclusion. $\amalg B$ adjudicated disagreements between reviewers (study selection and quality). KEAB, JOF, NKJA, JAP, and LJB prepared the initial and subsequent drafts of the manuscript, and integrated comments into revised versions of the manuscript. JOF double-checked data entry. All authors revised and approved the final version of the manuscript.

\section{Competing interests}

All authors declare: (1)no support from any organization for the submitted work; (2) no financial relationships with any organizations that might have an interest in the submitted work in the previous 3 years; and (3) no other relationship or activities that could appear to have influence the submitted work.

LJB's laboratory received equipment and/or research grants from Maquet (research on NAVA), Covidien (PAV), Philips (sleep), Fisher Paykel (high flow), Air Liquide (CPR), and General Electric (lung volume); and LJB received lecture fees from Covidien and Maquet.

\section{Consent for publication}

Not applicable.
Ethics approval and consent to participate

Not applicable.

\section{Sources of direct financial support}

None.

\section{Publisher's Note}

Springer Nature remains neutral with regard to jurisdictional claims in published maps and institutional affiliations.

\section{Author details}

${ }^{1}$ St Michael's Hospital and the Keenan Research Centre/Li Ka Shing Knowledge Institute, 30 Bond Street, Toronto, ON M5B 1W8, Canada. ${ }^{2}$ Interdepartmental Division of Critical Care, University of Toronto, Toronto, ON, Canada. ${ }^{3}$ Department of Clinical Epidemiology and Biostatistics, McMaster University, Hamilton, ON, Canada. ${ }^{4}$ Department of Critical Care Medicine and Sunnybrook Research Institute, Sunnybrook Health Sciences Centre, Toronto, ON, Canada. ${ }^{5}$ Department of Public Health, University of Toronto, Toronto, ON, Canada. ${ }^{6}$ Division of Critical Care of Moinhos de Vento Hospital, Porto Alegre, Brazil. ${ }^{7}$ Division of Critical Care of Hospital de Clínicas de Porto Alegre, Porto Alegre, Brazil.

Received: 3 February 2017 Accepted: 2 May 2017

Published online: 01 June 2017

\section{References}

1. Esteban A, Alia I, Ibanez J, et al. Modes of mechanical ventilation and weaning. A national survey of Spanish hospitals. The Spanish Lung Failure Collaborative Group. Chest. 1994;106:1188-93.

2. Esteban A, Anzueto A, Frutos F, et al. Characteristics and outcomes in adult patients receiving mechanical ventilation: a 28-day international study. JAMA. 2002;287(3):345-55.

3. Ely EW, Baker AM, Dunagan DP, et al. Effect on the duration of mechanical ventilation of identifying patients capable of breathing spontaneously. N Engl J Med. 1996:335:1864-9.

4. Blackwood B, Burns K, Cardwell C, et al. Use of weaning protocols for reducing duration of mechanical ventilation in critically ill adult patients: an updated Cochrane systematic review and meta-analysis. Cochrane Database Syst Rev. 2014;11:CD006904.

5. Kuhlen R, Max M, Dembinski R, et al. Breathing pattern and workload during automatic tube compensation, pressure support and T-piece trials in weaning patients. Eur J Anaesthesiol. 2003;20(1):10-6.

6. Oulette $D R$, et al. Liberation from mechanical ventilation in critically ill adults: an Official American College of Chest Physicians/American Thoracic Society Clinical Practice Guideline. Inspiratory pressure augmentation during spontaneous breathing trials, protocols minimizing sedation, and noninvasive ventilation immediately after extubation. Chest. 2017:151(1):166-80.

7. Ladeira MT, Vital FM, Andriolo RB, et al. Pressure support versus T-tube for weaning from mechanical ventilation in adults. Cochrane Database Syst Rev. 2014;5:CD006056

8. Dickersin K, Scherer R, Lefebvre C. Identifying relevant studies for systematic reviews. BMJ. 1994;309(6964):1286-91.

9. Lefebvre C, Manheimer E, Glanville J. Chapter 6: Searching for studies. In: Cochrane Handbook for Systematic Reviews of Interventions Version 5.0.1 (updated September 2008). The Cochrane Collaboration. 2011. www. cochrane-handbook.org. Accessed 16 May 2017.

10. Robinson KA, Dickersin K. Development of a highly sensitive search strategy for the retrieval of reports of controlled trials using PubMed. Int J Epidemiol. 2002;31(1):150-3.

11. Higgins JPT, Green S, editors. Cochrane Handbook for Systematic Reviews of Interventions Version 5.0.0. The Cochrane Collaboration. 2008. http:// handbook.cochrane.org. Accessed 16 May 2017.

12. Review manger (RevMan) Version 5.3.0. The Cochrane Collaboration. Copenhagen: The Nordic Cochrane Centre; 2011.

13. Higgins JP, Thompson SG. Quantifying heterogeneity in a meta-analysis. Stat Med. 2002;21:1539-58

14. Higgins JPT, Green S (editors). Cochrane Handbook for Systematic Reviews of Interventions Version 5.1.0 (updated March 2011). The Cochrane Collaboration. 2011. http://handbook.cochrane.org. Accessed 16 May 2017.

15. Borenstein M, Higgins JP. Meta-analysis and subgroups. Prev Sci. 2013;14:134-43. 
16. Guyatt $G$, Oxman AD, Kunz R, for the GRADE Working Group, et al. What is "Quality of Evidence" and why is it important to clinicians? BMJ. 2008;336:995-8.

17. Egger M, Davey Smith G, Schneider M, et al. Bias in meta-analysis detected by a simple, graphical test. BMJ (Clinical Research Ed). 1997; 315(7109):629-34

18. Feeley TW, Saumarez R, Klick JM, et al. Positive end-expiratory pressure in weaning patients from controlled ventilation. Lancet. 1975;2(7938):725-8.

19. Hastings PR, Bushnell L, Skillman JJ, et al. Cardiorespiratory dynamics during weaning with IMV versus spontaneous ventilation in good-risk cardiacsurvey patients. Anesthesiology. 1980;53:429-31.

20. Prakash O, Meij S, Van Der Borden B. Spontaneous ventilation test vs intermittent mandatory ventilation. Chest. 1982;81:403-6.

21. Koller W, Spiss C, Fina U, Duma S. Entwohnung nach postoperativer Beatmung Kardiochirurgischer Patienten, CPAP versus ZEEP [German]. Der Anesthesist. 1983:32:483-7.

22. Jones DP, Byrne P, Morgan C, et al. Positive end-expiratory pressure vs. T-piece. Extubation after mechanical ventilation. Chest. 1991;100:1655-59.

23. Abalos A, Leibowitz AB, Distafanco $D$, et al. Myocardial ischemia during the weaning period. Crit Care. 1992;3:32-6.

24. Bailey CR, Jones RM, Kelleher AA. The role of continuous positive airway pressure during weaning from mechanical ventilation in cardiac surgica patients. Anaesthesia. 1995;50:677-81.

25. Schinco MA, Whitman GJR, Weiman DS, et al. Pressure support ventilation in combination with continuous positive airway pressure is a better weaning trial in the post coronary bypass patient. Crit Care Med. 1995;23:A236.

26. Esteban A, Alia I, Gordo F, et al. Extubation outcome after spontaneous breathing trials with T-tube or pressure support ventilation. Am J Respir Crit Care Med. 1997;56:459-65.

27. Holanda MA, Rocha EM, Bandeira RM, et al. Pressure support ventilation (PSV) versus T-tube as pre-extubation spontaneous breathing trials (SBT) Am J Respir Crit Care Med. 2000;161:A559.

28. Farias JA, Retta A, Olazarri F, et al. A comparison of two methods to perform a breathing trial before extubation in pediatric intensive care patients. Intensive Care Med. 2001:27:1649-54.

29. Haberthur C, Mols G, Elsasser S, et al. Extubation after breathing trials with automatic tube compensation, T-tube, or pressure support ventilation. Acta Anaesthesiol Scand. 2002;46:973-9.

30. Koksal GM, Sayilgan C, Sen O, et al. The effects of different weaning modes on the endocrine stress response. Crit Care. 2004;8:R31-4.

31. Matic I, Majeric-Kogler V. Comparison of pressure support and T-tube weaning from mechanical ventilation: randomized prospective study. Croat Med J. 2004:45:162-6.

32. Cohen JD, Shapiro M, Grozovski E, et al. Extubation outcome following a spontaneous breathing trial with automatic tube compensation versus continuous positive airway pressure. Crit Care Med. 2006;34:682-6.

33. Liang CY, Chen CW, Lin WJ, et al. A prospective, randomized comparison of automated tube compensation (ATC) and T-piece in the weaning of mechanically ventilated patients. Am J Respir Crit Care Med. 2006;173:A41.

34. Colombo T, Boldrini AF, Juliano SRR, et al. Implementation, assessment and comparison of the T-tube and pressure-support weaning protocols applied to the intensive care unit patients who had received mechanical ventilation for more than 48 hours [Portugese]. Rev Bras Ter Intensiva. 2007;19:31-7.

35. Matic I, Danic D, Majeric-Kogler V, et al. Chronic obstructive pulmonary disease and weaning of difficult-to-wean patients from mechanical ventilation: randomized prospective study. Croat Med J. 2007:48:51-8

36. Fayed AM, El Feky IL. Adding automatic tube compensation to continuous positive airway pressure in weaning patients with chronic obstructive pulmonary disease, is it worth trying? Crit Care Med. 2008;36:A137.

37. Cohen J, Shapiro M, Grozovki E, et al. Prediction of extubation outcome: a randomized, controlled trial with automatic tube compensation vs. pressure support ventilation. Crit Care. 2009;13:R21.

38. Zhang B, Qin YZ. A clinical study of rapid-shallow breathing index in spontaneous breathing trial with pressure support ventilation and T-piece [Chinese]. Chinese Crit Care Med. 2009;21:397-401.

39. Figueroa-Casas J, Montoya R, Arzabala A, et al. Comparison between automatic tube compensation and continuous positive airway pressure during spontaneous breathing trials. Respir Care. 2010;55:549-54.

40. Molina-Saldarriaga FJ, Fonseca-Ruiz NJ, Castro C, et al. Study of spontaneous breathing in patients with chronic obstructive pulmonary disease: continuous positive airway pressure (CPAP) versus T-Tube [Portuguese]. Med Intensiva. 2010;34:453-8.
41. Cekman N, Erdemli O. The comparison of the effects of T-piece and CPAP on hemodynamic parameters, arterial blood gases and success of weaning. Bratisl Lek Listy. 2011;112:512-16.

42. Vats N, Singh J, Kaira S. Extubation outcome after spontaneous breathing trials with T-tube or pressure support ventilation. Indian J Physiother Occup Ther. 2012:6:86-9.

43. El-beleidy ASE, Khattab AAE, El-Sherbini SA, et al. Automatic tube compensation versus pressure support ventilation and extubation outcome in children; a randomized controlled study. ISRN Pediatr. 2013;871376:1-6.

44. Lourenco IS, Franco AM, Bassetto $S$, et al. Pressure support ventilation versus spontaneous breathing with T-tube for interrupting the ventilation after cardiac operations. Rev Bras Cir Cardiovasc. 2013;28:455-61.

45. Sherif AA, Atalaah HA. Prediction of weaning outcome, feasibility of automatic tube compensation (ATC) for weaning of chronic obstructive pulmonary disease (COPD) patients from mechanical ventilation. Anesth Analg. 2013;116:S-108.

46. Bilan N, Ganji S. Comparison of CPAP with humidifier, blender, and T-piece on the outcome of weaning in patients with neurologic disorders. Iran J Child Neurol. 2015:9:42-5.

47. Chittawatanarat K, Orrapin S, Orrapin S. An open label randomized control trial between low pressure support and T-piece method for discontinuation from mechanical ventilation and extubation in general surgical intensive care units. Crit Care. 2015;19:P268.

48. Teixeira SN, Osaku EF, Costa CRLM, et al. Comparison of proportional assist ventilation plus, T-tube ventilation and pressure support ventilation as spontaneous breathing trials for extubation: a randomized study. Respir Care. 2015;60:1527-35

49. Zhang B, Qin YZ. Comparison of pressure support ventilation and T-piece in determining rapid shallow breathing index in spontaneous breathing trials. Am J Med Sci. 2014:348:300-5.

50. Bilan N, Gangi S. Weaning from ventilator and effect of blender-humidifier on outcome. Int J Pediatr. 2014:2 Suppl 6:39-45.

51. Pellegrini JAS, Moraes RB, Maccari JG, et al. Spontaneous breathing trials with T-piece or pressure-support ventilation: a systematic review and metaanalysis of RCTs. Respir Care. 2016;61(12):1693-703.

52. Brochard L, Rauss A, Benito S, et al. Comparison of three methods of gradual withdrawal from ventilatory support during weaning from mechanical ventilation. Am J Respir Crit Care Med. 1994;150:896-903.

53. Esteban A, Frutos F, Tobin MJ, et al. A comparison of four methods of weaning patients from mechanical ventilation. N Engl J Med. 1995;332:345-50.

54. Koh Y, Hong SB, Lim CM, et al. Effect of an additional 1-hour T-piece trial on weaning outcome at minimal pressure support. J Crit Care. 2000;15:41-5.

55. Vitacca M, Vianello A, Colombo D, et al. Comparison of two methods for weaning patients with chronic obstructive pulmonary disease requiring mechanical ventilation for more than 15 days. Am J Respir Crit Care Med. 2001;164:225-30.

56. Jubran A, Grant BJ, Duffner LA, et al. Effect of pressure support vs unassisted breathing through a tracheostomy collar on weaning duration in patients requiring prolonged mechanical ventilation: a randomized trial. JAMA. 2013;309(7):671-7

57. Sklar MC, Burns K, Rittayamai N, et al. Effort to breathe with various spontaneous breathing trial techniques: a systematic review and physiological meta-analysis. Am J Respir Crit Care Med. 2016.[Epub ahead of print].

58. Tobin MJ, Jubran A. Variable performance of weaning-predictor tests: role of Bayes' theorem and spectrum and test-referral bias. Intensive Care Med. 2006;32:2002-12.

59. Thille AW, Richard JC, Brochard L. The decision to extubate in the intensive care unit. Am J Respir Crit Care Med. 2013;187:1294-302.

60. Afessa B, Hogans L, Murphy R. Predicting 3-day and 7-day outcomes of weaning from mechanical ventilation. Chest. 1999;116:456-61.

61. Stroetz RW, Hubmayr RD. Tidal volume maintenance during weaning with pressure support. Am J Respir Crit Care Med. 1995;152:1034-40.

62. Burns KEA, Karottaiyamvelil Jacob S, Aguirre V, et al. Stakeholder engagement in trial design: a survey of citizen's preference for outcomes and treatment options during weaning. Ann Am Thorac Soc. 2016;13(11):1962-8.

63. Esteban A, Alia I, Tobin MJ, Gil A, Gordo F, Vallverdu I, Blanch L, Bonet A Vazquez A, de Pablo R, Torres A, de al Cal MA, Macias S, for the Spanish Lung Failure Collaborative Group. Effect of spontaneous breathing trial duration on outcome of attempts to discontinue mechanical ventilation. AJRCCM. 1999;159:512-8 
64. Perren A, Domenighetti G, Mauri S, Genini F, Vizzardi N. Protocol-directed weaning from mechanical ventilation: clinical outcome in patients randomized for a 30-min or 120-min trial with pressure support ventilation. Intens Care Med. 2002;28:1058-63.

65. Fernandez MM, Fernandez R, Magret M, González-Castro A, Bouza MT, Ibañez M, García C, Balerdi B, Mas A, Arauzo V, Añón JM, Ruis F, Ferreres J, Tomás R, Alabert M, Tizón Al, Altaba S, Llamas N. Reconnection to mechanical ventilation for one hour after a successful spontaneous breathing trial reduces extubation failure and reintubation in critically ill patients: a multicenter randomized controlled trial. Intens Care Med Exp. 2016:4 Suppl 1:A470.

Submit your next manuscript to BioMed Central and we will help you at every step:

- We accept pre-submission inquiries

- Our selector tool helps you to find the most relevant journal

- We provide round the clock customer support

- Convenient online submission

- Thorough peer review

- Inclusion in PubMed and all major indexing services

- Maximum visibility for your research

Submit your manuscript at www.biomedcentral.com/submit
Biomed Central 